\title{
Macro- and microstructural descriptions of the zebrafish (Danio rerio) liver
}

\author{
L. Batelli de Oliveira, A. Santos Leonardo, E.M. Mendes de Lima \\ Laboratory of Veterinary Anatomy, Veterinary Hospital, University of Brasilia, Brazil
}

[Received: 17 December 2015; Accepted: 5 February 2016]

The liver is an essential metabolically active organ, responsible for many vital life functions such as metabolism, synthesis, and storage. This study describes the macroscopic and microscopic zebrafish liver and its temporal changes during a period of 13 weeks. Forty-six adult zebrafish were used (46), of which 27 were females and were 19 males. Seven fish were euthanized initially for macroscopic evaluation and 3 fish were euthanized weekly for 13 weeks for microscopic evaluation. Routine histological technique was performed; the slides were stained with haematoxylin/eosin and periodic acid-Schiff. The volumetric $(V v(h))$ and numeri$\mathrm{cal}(\mathrm{Nv}(h))$ densities of hepatocytes and vacuolation intensity were determined weekly. The liver was located in the coelomic cavity ventrally to the swim bladder, slightly brownish and divided into 3 hepatic lobes. Microscopically, it consisted of hepatocytes forming a muralium duplex around the sinusoids. The staining of the cytoplasm of hepatocytes was more eosinophilic and more basophilic in males and females, respectively. There was no formation of portal triads and phagocytic cells. The gallbladder had transitional epithelium, and the bile ducts had simple cuboidal epithelium. The volume and numerical density of hepatocytes during the 13 weeks was not statistically different, but these parameters displayed a positive correlation. The vacuolation was due to the presence of glycogen. The zebrafish liver has macro and micro structural particular features while, quantitatively, the volume and number of hepatocytes showed great capacity to respond to the physiological needs of the animal at the time. (Folia Morphol 2016; 75, 3: 382-387)

Key words: hepatocytes, fish density, macroscopy, glycogen

\section{INTRODUCTION}

The fish liver, as well as in other vertebrates, is the largest gland in the body and is responsible for many vital life functions and animal physiology. The liver is located in the coelomic cavity and is made up of hepatocytes, cells with high potential mitotic whose function is synthesis, secretion, storage, biotransformation, and metabolism [2].

In general, hepatocytes metabolise endogenous and exogenous substances; produce bile, which is subsequently directed to the gallbladder, and essen- tial proteins of the plasma and coagulation factors; and store substances such as amino acids, lipids, glycogen, and iron [7].

Moreover, another important role of the liver in fish is that it serves as an indicator of their nutritional and physiological status [1]. Due to the metabolism functions, it also serves as environmental pollution bioindicator in this species [5].

Therefore, this study described zebrafish liver macroscopically and microscopically and assessed temporal changes over a period of 13 weeks due to 
the liver relevance to maintain the homeostasis of the species.

\section{MATERIALS AND METHODS}

The trial protocol was approved by the Ethics Committee on Animal Experimentation of the Universidade de Brasilia under Protocol No. 127542/2013.

A total of 46 Danio rerio, known as zebrafish (27 females and 19 males) aged between 12 and 18 months (adults) were used in the trial. The animals remained in quarantine and acclimatisation in a 19-L aquarium for about 60 days. The population density was 3 fish per litre. The experiment lasted 13 weeks so that fish age at the end of the experiment was between 16 and 22 months (adults) corresponding to approximately $48 \%$ of the average life expectancy in the laboratory [3].

During the adaptation period and experiment, the animals were submitted to a cycle of $14 \mathrm{~h}$ of light and $10 \mathrm{~h}$ of darkness and fed commercial diet twice daily (alcon Basic ${ }^{\circledR}$ ). The fish tank was equipped with a closed loop system at a thermostatically controlled temperature of $26 \pm 2^{\circ} \mathrm{C}\left(\right.$ Atman $\left.^{\circledR}\right)$.

The following water quality parameters $\mathrm{pH}(\mathrm{pH}$ Tropical, LabconTest), ammonia (toxic ammonia, LabconTest), and dissolved oxygen (Dissolved $\mathrm{O}_{2}$, LabconTest) were tested daily, weekly, and monthly, averaging $6.8-7.0 \mathrm{mg} / \mathrm{L}, 0-0.001 \mathrm{mg} / \mathrm{L}$, and $6-8 \mathrm{mg} / \mathrm{L}$, respectively. The temperature was also measured daily using a glass thermometer (Aquadene ${ }^{\circledR}$ ).

Animals were euthanized by immersion in an aqueous solution with $0.2 \%$ 3-ethyl-aminobenzoate (Sigma-Aldrich Chemistry), followed by immersion in ice water $\left(4^{\circ} \mathrm{C}\right)$ for $15 \mathrm{~min}[4]$.

For the macroscopic evaluation, 7 randomly chosen fish were euthanized, and a necropsy was performed according to the techniques described for dissecting zebrafish [4]. Fish liver was examined using an optical microscope (BX51 Olympus ${ }^{\circledR}$ ) with objective of $4 \times$. The images were captured (BMP, 24-bit colour, $2048 \times 1536$ pixels) by a camera coupled (Moticam 3, Motic $\left.^{\circledR}\right)$ to the microscope and analysed using the image analysis software (ProgRes ${ }^{\circledR}$ Capture Pro 2.5).

For microscopic evaluation, 3 fish were euthanized weekly. The fish cadavers were fixed in Dietrich aqueous solution for $24 \mathrm{~h}$. Only the operculum and the left pectoral and caudal, fins were removed, as recommended by the Zebrafish International Resource Centre (ZIRC). The routine histological technique was used, dehydration through a series of increasing etha- nol concentrations, diaphanisation in xylene, impregnation and paraffin embedding. The blocks were cut in the sagittal plane into $3 \mu \mathrm{m}$-thick sections using a manual microtome (Leica RM 2125RT). From each fragment, 3 blades were prepared with at least 2 cuts in each. The liver tissue cuts were stained with haematoxylin/eosin for histological examination. The following parameters were checked: the organisation of the hepatic parenchyma, topography, cell conformation and organ components. In addition, the cuts were also stained by the periodic acid-Schiff (PAS) method.

Nine random field images were captured per week using the optical microscope (Leica DM500 ${ }^{\circledR}$ ) coupled to a camera (Leica ICC50 HD ${ }^{\circledR}$ ) and analysed by image analysis software (LAS EZ Leica ${ }^{\circledR}$, type 2.0). The volumetric $(\mathrm{Vv}(\mathrm{h}))$ and numerical $(\mathrm{Nv}(\mathrm{h}))$ density of hepatocytes was determined. The intensity of vacuolation per week was also evaluated and scored using a cross-system that ranged from one cross $(+)$ less intense vacuolation to three crosses $(+++)$ indicating greater vacuolation [6].

The volumetric densities were determined by counting the points that touched the nucleus of hepatocytes. The numerical density of hepatocytes was obtained from the number of nuclei viewed per area. The stereological analysis software STEPanizer ${ }^{\circledR}$ (http://stepnizer.com/) was used to quantify the numerical and volumetric densities.

The weekly data were subjected to descriptive statistics to obtain the mean and standard error of the mean, followed by the Kolmogorov-Smirnov normality test, analysis of variance one-way ANOVA and Pearson or Spearman correlation, depending on the result of the normality test. All tests used the GraphPad Prism ${ }^{\circledR} 6$ software at $\mathrm{p} \leq 0.05$ significance level.

\section{RESULTS}

\section{Macroscopic description}

The liver is located in the coelomic cavity as a parenchymal organ with dense and homogeneous aspect, slightly brownish and rather vascularised, in the medial body region (Fig. 1A). The ventral coelomic wall was observed ventrally to the liver, or depending on the distension, to the intestine. The intestine was also located medially to the liver along with the pancreas (Fig. 1B). Also, the gallbladder was observed in the ventral portion of the liver, as an oval and green structure with a sacculation shape (Fig. 1C). The swim bladder was observed dorsally and the 

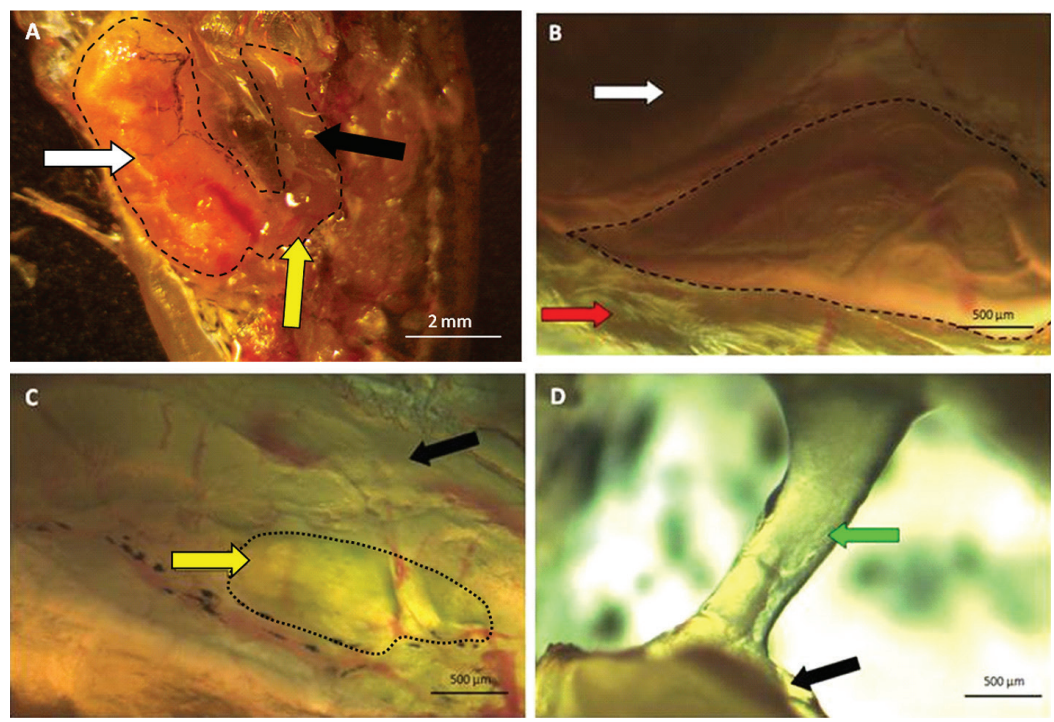

Figure 1. Zebrafish liver; A. Left hepatic lobe (dashed) highly vascularised (thin arrow); B. Intestine (white arrow) and ventral coelomic wall (red arrow); C. Left liver lobe (black arrow) with gallbladder (yellow arrow and dotted line); D. Transverse lobe (green arrow).

reproductive organs (ovary or testis), caudally. The kidney was located cranially to the liver, in the most dorsal portion, and venous sinus, ventral. Overall, the liver appeared to be made up of three asymmetric, hepatic lobes, two lateral (right and left) with a bigger left one, and a smaller transverse one that united the lateral lobes (Fig. 1D).

\section{Histological description}

The histological section showed that the liver and the other organs were located in the coelomic cavity (Fig. 2A) and consisted mainly of hepatocytes arranged in 2 cellular layer and surrounded by sinusoids (Fig. 2B). The hepatocytes were polyhedral, with distinct edges, broad cytoplasm with different degrees of vacuolation. The cytoplasmic staining differed between the sexes, being more eosinophilic in males (Fig. 2C) and more basophilic, in females (Fig. 2D). The nucleus of the hepatocytes was single, round, basophilic and, generally, central or slightly peripheral. In the cases where vacuolation was more accentuated, nucleus was displaced towards the edge (Fig. 2G). There was no formation of a portal triad or presence of phagocytic cells.

The gallbladder was presented as a hollow structure, with transitional epithelium, while the bile ducts had simple cuboidal epithelium (Fig. 2E).

The blood vessels of the liver were made up of simple squamous epithelium, similar to that observed in the liver capsule that covers the body (Fig. 2G).

\section{Temporal analysis}

The volume and numerical density of hepatocytes and vacuolation intensity over the 13 weeks are shown in Figure 3 and Table 1. The correlation tests among the parameters evaluated are shown in Table 2.

PAS staining was positive (Fig. 4A, B), indicating that the observed vacuolation was glycogen degeneration.

Most weeks showed a significant positive correlation between volume and numerical densities of hepatocytes varying from moderate to strong. These variables were independent only in the $2^{\text {nd }}$ and $11^{\text {th }}$ weeks since the statistical difference showed no correlation.

\section{DISCUSSION}

\section{Macroscopy and histology}

The macroscopic and microscopic findings observed in zebrafish liver were similar to those found in other teleosts $[1,2,4]$. The liver location in the medial portion of the coelomic cavity, its size and shape were due to an adjustment to the space occupied by other organs during the embryonic development [2] . Moreover, the brownish colour resulted from the rich vasculature of the organ.

Unlike mammals, absence of division into hepatic lobules and phagocytic cells are features of zebrafish [7]. Furthermore, they formed a muralium duplex of cells surrounding a sinusoidal capillary. 

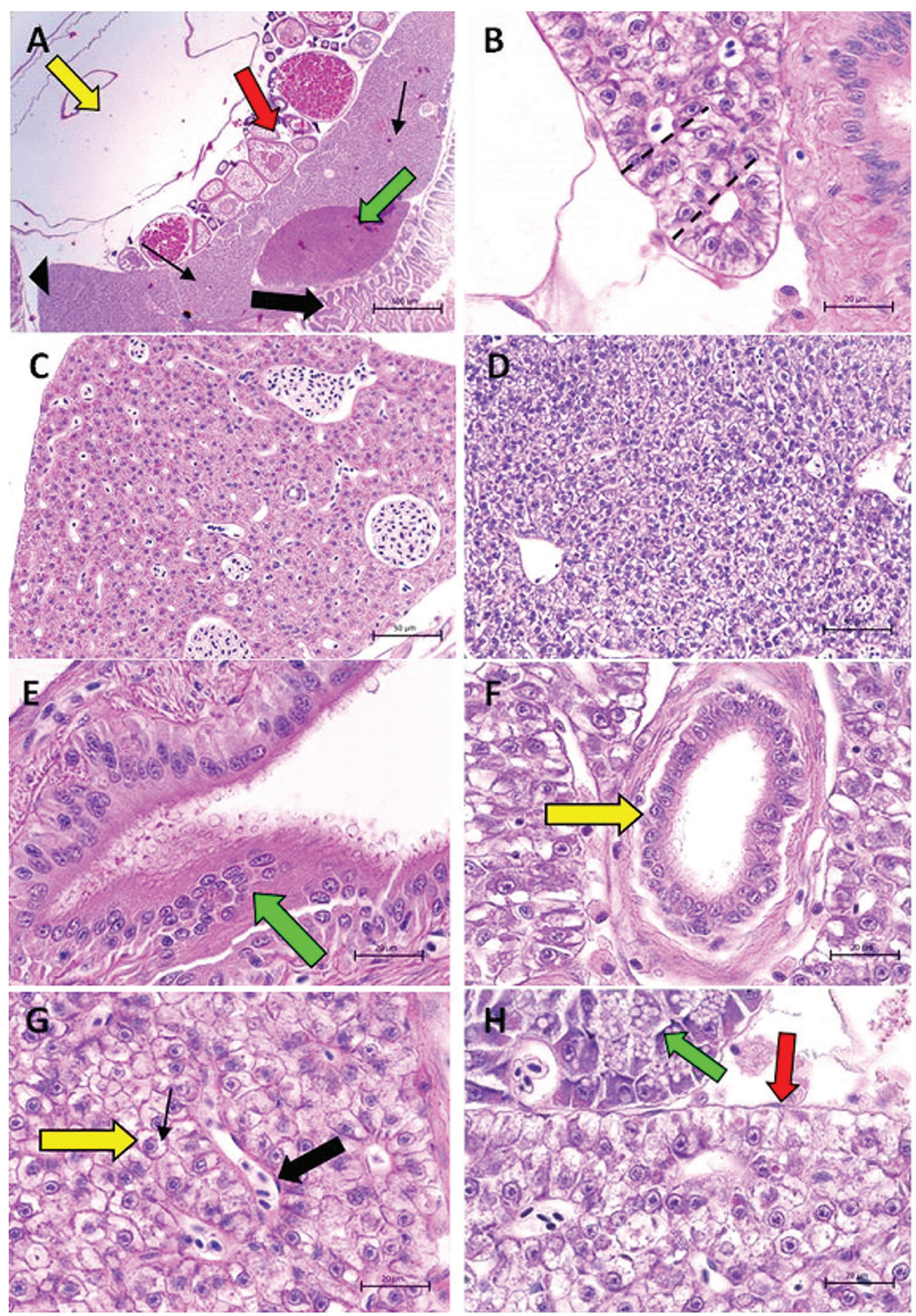

Figure 2. Zebrafish liver stained with haematoxylin/eosin; A. Swim bladder (thick yellow arrow), ovary (thick red arrow), liver (thin arrows), pancreas (thick green arrow), intestine (thick black arrow) and kidney (arrowhead); B. Muralium duplex of hepatocytes around the sinusoids (dashed); C. Eosinophilic staining of male liver; D. Basophilic staining of female liver; E. Gallbladder (thick green arrow); F. Cuboidal epithelium of the bile duct (thick yellow arrow); G. Squamous epithelium of the blood vessel (thick black arrow), hepatocyte with nucleus (yellow arrow) and vacuolation (thin arrow); H. Epithelium of the coating capsules (thick red arrow) and pancreas (thick green arrow).
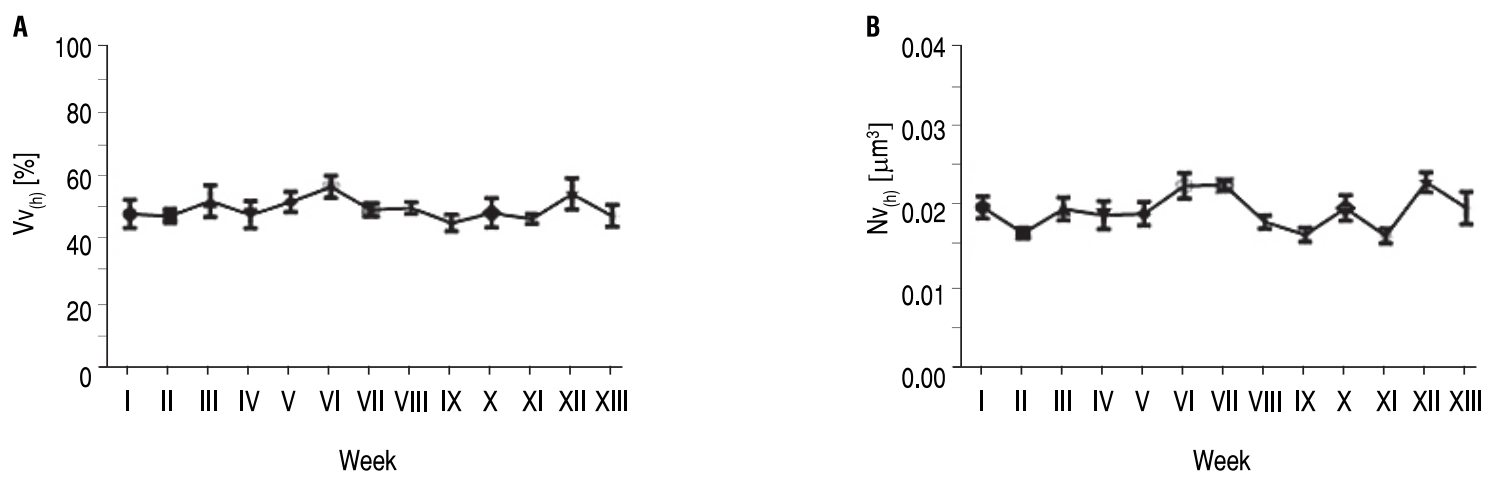

Figure 3. Plot of the mean \pm standard error values of density during the 13-week period; A. Volumetric density of hepatocytes $-\mathrm{Vv}(\mathrm{h})$; B. Numerical density of hepatocytes — Nv(h). There were no statistically significant differences over the 13-week period. One-way ANOVA. 
Table 1. Variation of the vacuolation over the 13-week period

\begin{tabular}{lccccccccccccc}
\hline Week: & I & II & III & IV & V & VI & VII & VIII & IX & X & XI & XII & XIII \\
\hline Vacuolisation & + & +++ & ++ & ++ & +++ & ++ & +++ & +++ & ++ & +++ & ++ & + & + \\
\hline
\end{tabular}

$(+)$ discreet; $(++)$ moderate; $(+++)$ accentuated

Table 2. Pearson or Spearman correlation $(r)$ of the volume $\left(\mathrm{Vv}_{(\mathrm{h})}\right)$ and numerical $\left(\mathrm{Nv}_{(\mathrm{h})}\right)$ density of hepatocytes over the 13-week period. Statistically significant r-values $\left({ }^{*}\right)$

\begin{tabular}{|c|c|c|c|c|c|c|c|c|c|c|c|c|c|}
\hline Week: & $\mathbf{I}$ & II & III & IV & V & VI & VII & VIII & IX & $x$ & XI & XII & XIII \\
\hline $\begin{array}{l}\text { r-values } \\
\text { Vv(h) } \times N v(h)\end{array}$ & $0.86^{*}$ & 0.04 & $0.94^{*}$ & $0.93^{*}$ & $0.69^{*}$ & $0.69^{*}$ & 0.38 & $0.75^{*}$ & $0.66^{*}$ & $0.75^{*}$ & 0.06 & 0.61 & $0.88^{*}$ \\
\hline
\end{tabular}

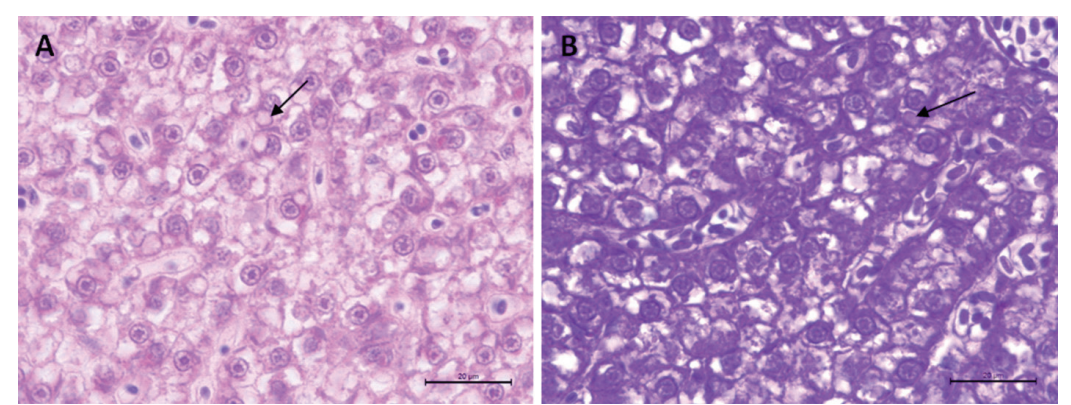

Figure 4. Zebrafish liver on the $10^{\text {th }}$ week; A. Vacuolation (arrow) intensity $(++)$ by haematoxylin/eosin staining; B. Vacuolation (arrow) intensity $(++)$ positive for periodic acid-Schiff.

This arrangement has been reported before in Oreochromis niloticus and Leporinus macrocephalus $[1,11]$. The absence of triad and phagocytic cells suggested that the liver in this species has evolved less compared with mammals. The muralium duplex suggests a mechanism for facilitating the nutrition of hepatocytes and releasing of their products into the bloodstream.

A species-specific characteristic of the zebrafish was the ability to differentiate genders by staining of the cytoplasm of hepatocytes. The females had basophilic cytoplasm while the males, eosinophilic. This basophilic staining observed in females resulted from the vitellogenin production [7]. The vitellogenin was characterised as a fat-phospho-glycoprotein produced in the liver of females by hepatocytes due to estradiol stimulation. It is released into the bloodstream and taken up by the oocyte to give rise to the egg yolk proteins that keep the embryo [8]. Therefore, this study suggests that females had higher amounts of vitellogenin in the hepatocytes compared with males. Probably this was observed because vitellogenesis occurred before the reproduction period.

Another particular feature of zebrafish was the transitional epithelium of the gallbladder. This type of epithelium was also observed in other zebrafish [7]. The transitional epithelium has a great ability to stretch and distort, allowing the organ volume to increase and decrease without leaking the contents. On the other hand, in the prismatic, each cell has a function such as water absorption and ion exchange to concentrate the bile. Therefore, it was concluded that the gallbladder in this species only stores and does not concentrate the bile, and that the bile is being excreted as it was produced by hepatocytes.

\section{Temporal analysis}

The volume and numerical density of hepatocytes along the 13 weeks showed no significant differences. Otherwise, there was a positive correlation between them varying from moderate to strong and significant for the most part. This finding indicated that factors which affected the volumetric density also interfered with the numerical density. The increasing demand for protein to produce vitellogenin and other hepatic precursors, especially in the breeding season, resulted in the proliferation of the rough endoplasmic reticulum and dictyosome [10]. Hepatocyte proliferation was observed during the breeding season in Salmo trutta fario, followed by a decrease in the number 
of these cells caused by an alternating mitosis and apoptosis cycle in the liver. These changes were found, both in males and in females, but it was more intense in females than in males [9]. Therefore, in this study, it is suggested that the changing volumetric density of hepatocytes in zebrafish resulted from the proliferation of organelles, and the changing numerical density is due to mitosis and apoptosis according to the production demand, especially of protein. Furthermore, the metabolic demand showed a trend with the liver mass and, consequently, with the densities of hepatocytes.

The different vacuolation intensities observed in the animals of this experiment were positive in PAS immunohistochemistry, indicating glycogen degeneration. In a study with Oreochromis niloticus, hepatocytes with relatively low amounts of organelles were observed, thus suggesting a low activity to secrete proteins compared with mammals and justifying its intracellular accumulation [11]. There are studies reporting that the amount of glycogen was related to the nutritional status and that well fed and healthy animals had higher glycogen content compared with those with altered liver [10]. Therefore, in this study, the diffuse presence of intracytoplasmic glycogen, albeit with different levels, indicated that zebrafish were well nourished and healthy during the studied period. Its accumulation also led to believe that this species has a low amount of organelles that would help in excretion.

The same glycogen variability has been reported for Salmo trutta fario, which had a high content, especially in the reproductive period because there is great energy demand at this time to produce protein and synthesize lipid [10]. Finally, it was concluded that many factors interfere with glycogen levels because this content has been linked to energy production, suggesting that its demand depends on the physiological needs of the fish, especially during the evaluated time.

\section{CONCLUSIONS}

The morphological characteristics of the zebrafish liver were similar to those in other teleost, differing regarding the histologic organisation that was characterised by a bilayer surrounding the sinusoids and absence of liver triads. Histochemical analysis allowed differentiating the liver of male and female zebrafish, suggested by the presence of vitellogenin in females. The volume and numerical density of hepatocytes was not significantly different during the 13-week period. However, the quantitative correlation between these parameters was significant and positive in most weeks, indicating that the liver has great plasticity regarding the structural and functional changes, adapting in volume and number according to the physiological needs.

\section{REFERENCES}

1. Bombonato MTS, Rochel SS, Vincentini CA, Vicentini IBF (2007) Estudo morfológico do tecido hepático de Leporinus macrocephalus. Acta Sci Biol Sci, 29: 81-85.

2. Costa GM, Ortis RC, Lima MG, Casals JB Lima AR, Jr Kfoury JR (2012) Estrutura morfológica do fígado de tambaqui Colossoma macropomum (Cuvier, 1818). Pesq Vet Bras, 32: 947-950.

3. Gilbert MJ, Zerulla TC, Tierney KB (2014) Zebrafish (Danio rerio) as a model for the study of aging and exercise: physical ability and trainability decrease with age. Exp Gerontol, 50: 106-113.

4. Gupta T, Mullins MC (2010) Dissection of organs from the adult zebrafish. JoVE, 37: e.1717.

5. Fredello JP, Raqbi A, Mattei X, Viale D, Marchand B (2001) Quantification of macrophage aggregates in the liver of Mugil cephalus. J Submicr Cytol Pathol, 33: 473-473.

6. Li X, Elwell MR, Ryan AM, Ochoa R (2003) Morphogenesis of postmortem hepatocyte vacuolation and liver weight increases in Sprague-Dawley rats. J Toxicol Pathol, 31: 682-688.

7. Menke AL, Spitsbergen JM, Wolterbeek AP, Woutersen RA (2011) Normal anatomy and histology of the adult zebrafish. Toxicol Pathol, 39: 759-775.

8. Pankhurst NW, Munday PL (2011) Effects of climate change on fish reproduction and early life history stages. Mar Freshwater Res, 62: 1015-1026.

9. Rocha E, Rocha MJ, Galante MH, Silva MW, Monteiro RAF (2009) The hepatocytes of the brown trout (Salmo trutta fario): a stereological study of their number and size along the breeding cycle. Ichthyol Res, 56: 43-54.

10. Rocha E, Rocha MJ, Lobo-da-cunha A, Galante MH, Monteiro RA (2010) The hepatocytes of the brown trout (Salmo trutta fario): a stereological study of some cytoplasmic components with the breeding cycle. Microsc Res Tech, 73: 766-778.

11. Vicentini CA, Franceschini-Vicentini IB, Bombonato MTS, Bertolucci B, Lima SG, Santos AS (2005) Morphological study of the liver in the teleost Oreochromis niloticus. Int J Morphol, 23: 211-216. 